\title{
Histopathologic Evaluation of Nonalcoholic Fatty Liver Disease in Hypothyroidism-Induced Rats
}

\author{
Şule Demir, ${ }^{1}$ Mustafa Ünübol, ${ }^{2}$ Serap Ünübol Aypak, ${ }^{3}$ Emrah İpek, ${ }^{4}$ Serdar Aktaş, ${ }^{5}$ \\ Gamze Sevri Ekren, ${ }^{3}$ Murat Yılmaz, ${ }^{6}$ Recai Tunca, ${ }^{4}$ and Engin Güney ${ }^{2}$ \\ ${ }^{1}$ Department of Internal Medicine, Adnan Menderes University Faculty of Medicine, 09100 Aydin, Turkey \\ ${ }^{2}$ Division of Endocrinology, Department of Internal Medicine, Adnan Menderes University Faculty of Medicine, 09100 Aydin, Turkey \\ ${ }^{3}$ Department of Biochemistry, Adnan Menderes University Faculty of Veterinary Medicine, 09100 Aydin, Turkey \\ ${ }^{4}$ Department of Pathology, Adnan Menderes University Faculty of Veterinary Medicine, 09100 Aydin, Turkey \\ ${ }^{5}$ Department of Pharmacology Toxicology, Adnan Menderes University Faculty of Veterinary Medicine, 09100 Aydin, Turkey \\ ${ }^{6}$ Department of General Surgery, Adnan Menderes University Faculty of Medicine, 09100 Aydin, Turkey
}

Correspondence should be addressed to Mustafa Ünübol; drmunubol@yahoo.com.tr

Received 8 December 2015; Accepted 17 March 2016

Academic Editor: Constantinos Pantos

Copyright (C) 2016 Şule Demir et al. This is an open access article distributed under the Creative Commons Attribution License, which permits unrestricted use, distribution, and reproduction in any medium, provided the original work is properly cited.

\begin{abstract}
It is speculated that thyroid hormones may be involved in nonalcoholic fatty liver disease (NAFLD) pathogenesis. A literature scan, however, demonstrated conflicting results from studies investigating the relationship between hypothyroidism and NAFLD. Therefore, our study aims to evaluate NAFLD, from the histopathologic perspective, in hypothyroidism-induced rats. Wistar rats were divided into 2 groups: the experimental group consumed water containing methimazole $0.025 \%$ (MMI, Sigma, USA) for 12 weeks and the control group consumed tap water. At the end of week 12, serum glucose, ALT, AST, triglyceride, HDL, LDL, TSH, fT4, fT3, visfatin, and insulin assays were performed. Sections were stained with hematoxylin-eosin and "Oil Red-O" for histopathologic examination of the livers. In our study, we detected mild hepatosteatosis in all hypothyroidism-induced rats. There was statistically significant difference with respect to obesity between the two groups $(p<0.001)$. The mean fasting blood glucose was $126.25 \pm 23.4 \mathrm{mg} / \mathrm{dL}$ in hypothyroidism-induced group and $102.63 \pm 15.51 \mathrm{mg} / \mathrm{dL}$ in the control group, with a statistically significant difference between the groups $(p=0.032)$. The two groups did not differ statistically significantly with respect to visfatin levels $(p>0.05)$. In conclusion, we found that hypothyroidism-induced rats had mild hepatosteatosis as opposed to the control group histopathologically. Our study indicates that hypothyroidism can cause NAFLD.
\end{abstract}

\section{Introduction}

Thyroid hormones have important roles in body weight, adipogenesis, energy homeostasis, and regulation of carbohydrate and lipid metabolism $[1,2]$. Clinical studies of hypothyroidism have shown a possible association with metabolic syndrome, obesity, and impaired lipid metabolism [3, 4]. Nonalcoholic fatty liver disease (NAFLD) is a chronic hepatic disease, with a broad histopathologic spectrum ranging from a simple steatosis to nonalcoholic steatohepatitis (NASH), fibrosis, and cirrhosis $[5,6]$. Closely associated with insulin resistance, hypertension, dyslipidemia, and obesity, NAFLD is believed to reflect the hepatic component of the metabolic syndrome [7].
It is speculated that thyroid hormones may be involved in NAFLD pathogenesis [8]. A literature scan, however, demonstrated conflicting results from studies investigating the relationship between hypothyroidism and NAFLD [913]. Chung et al. [13] reported that ultrasonographically determined NAFLD development was more frequent among patients with hypothyroidism compared to healthy controls. However, Ittermann et al. [12] identified no relationship between hepatosteatosis and hypothyroidism. In their article reviewing the studies on the relationship between thyroid dysfunction and NASH/NAFLD, Eshraghian and Jahromi [8] emphasized that subclinical hypothyroidism and hypothyroidism were more common in patients with NASH/NAFLD 
and that hypothyroidism was an independent risk factor for NASH/NAFLD in some studies, although some recent studies have reported no correlation. Hence, the need for new studies to elucidate the relationship between NAFLD and hypothyroidism was highlighted.

Therefore, our study aims to evaluate NAFLD, from the histopathologic perspective, in hypothyroidism-induced rats.

\section{Materials and Methods}

2.1. Ethics Board Approval. For the present study, ethics board approval of the Local Ethics Committee for Animal Experiments of Adnan Menderes University (ADÜ-HADYEK) was received on 27/01/2015 (decision number 64583101/2015/002).

2.2. Study Protocol. Sixteen adult male Wistar Albino rats (8-12 weeks, $250 \mathrm{~g}-330 \mathrm{~g}$ ) were used for this study. The rats were maintained at room temperature at $24^{\circ} \mathrm{C} \pm 1$, alternately for 12 hours under lit (07:00-19:00) and 12 hours at dark (19:00-07:00) conditions. All rats were monitored in the same environment and were fed with the same standard feed and were provided with water and feed ad libitum.

Group 1 (Healthy Control Group $(n=8)$ ). The rats in this group were only given daily drinking water and feed.

Group 2 (Hypothyroidism-Induced Group $(n=8)$ ). Hypothyroidism was induced by adding methimazole 0.025\% (MMI, Sigma, USA) to drinking water for 12 weeks [14].

Rats' bodyweights were measured weekly. At the end of week 12, bodyweights following a 12-hour fasting and nasoanal length were measured in the rats in all groups. Lee index was used to evaluate obesity. The index was calculated as follows: body weight $(\mathrm{g})$ cubic root $\times 10 /$ nasoanal length $(\mathrm{mm})$. Results $\leq 0.300$ were considered normal and results $>0.300$ were considered as obesity [15]. For anesthesia, intraperitoneal $40 \mathrm{mg} / \mathrm{kg}$ ketamine $+4 \mathrm{mg} / \mathrm{kg}$ xylazine was administered. Under anesthesia, blood was collected from systemic circulation and cervical dislocation was performed. The material for biochemical analysis was foil-wrapped and was transferred within a very short time to the biochemistry laboratory.

2.3. Serum and Blood Sample Preparation. Blood samples taken from all rats were collected in gel-containing serum tubes. Serum samples were divided into small portions in amounts sufficient for intended analyses and were kept at $-20^{\circ} \mathrm{C}$ pending analyses (except glucose and ALT analyses, which were performed immediately). Serum glucose, ALT, AST, triglyceride, HDL, LDL, and insulin assays were performed using the commercial kits of BIOLABO (Maizy, France). Immunoassay based on specific sandwich Elisa method was used to determine free triiodothyronine (fT3) (SunRed, Biotechnology Company, Shanghai, Cat. number 201-11-0738), free thyroxine (fT4) (SunRed, Biotechnology Company, Shanghai, Cat. number 201-11-0736), thyroid-stimulating hormone (TSH) (SunRed, Biotechnology Company,
Shanghai, Cat. number 201-11-0181), and visfatin (SunRed, Biotechnology Company, Shanghai, Cat. number 201-110472) levels in sera obtained from the rats. TSH kits had a measuring range of $0.03-6 \mathrm{mIU} / \mathrm{L}$, sensitivity of $0.021 \mathrm{mIU} / \mathrm{L}$, Intra-Assay CV of $<10 \%$, and Inter-Assay CV of $<12 \%$. sT 4 kits had a measuring range of $0.5-150 \mathrm{pmol} / \mathrm{L}$ and sensitivity of $0.466 \mathrm{pmol} / \mathrm{L}$.

2.4. Histopathologic Assessment. Livers were removed following euthanasia and were first washed with physiological saline solution. Recommendations of Ruehl-Fehlert et al. [16] were followed in trimming the livers and determining the sections to be examined. After washing, the liver was trimmed to include the left lateral lobe, caudate lob, and right and left medial lobe. The trimmed tissues were conveniently placed into cassettes and fixed in 10\% neutral buffered formalin (Sigma-Aldrich) for 24-48 hours, after which the tissues were washed with running tap water for 6-8 hours and were then embedded in paraffin using the automatic tissue processing instrument (Leica TP1020). Sections of paraffin-embedded tissues, $5 \mu \mathrm{m}$ thick, were transferred onto slides (Thermo Scientific). Sections were stained with hematoxylin-eosin for histopathologic examination. To demonstrate fat vacuoles in the liver, trimmed tissues were sliced in $10 \mu \mathrm{m}$ thickness with a freezing microtome. The sections were then stained using the "Oil Red-O" method. The obtained liver preparations were examined under light microscope (Olympus BX51) and microscopic digital photographs were taken (Olympus C5050), which were transferred into computer.

2.5. Statistical Analysis. Data obtained from the study were analyzed using the SPSS 17.0 (Statistical Package for the Social Science, version 17.0). For comparative analyses, distribution pattern was first determined using the Kolmogorov-Smirnov test and then Student's $t$-test and chi-square were used. $p$ values $<0.05$ were considered statistically significant.

\section{Results}

3.1. Obesity Assessment. Mean fasting bodyweight measured on the first day of the study was $287.25 \pm 15.78 \mathrm{~g}$ in the hypothyroidism group and $282.88 \pm 33.59 \mathrm{~g}$ in the control group, which were similar $(p>0.05)$. Mean fasting bodyweight measured on the last day of the study was $297.38 \pm 19.62 \mathrm{~g}$ in the hypothyroidism group and compared to $290.75 \pm$ $33.34 \mathrm{~g}$ in the control group, with no statistically significant difference $(p>0.05)$. With obesity assessment based on Lee index, baseline measurements were comparable between the groups $(p>0.05)$, while Lee indices at the end of the study were $0.309 \pm 0.005$ in the hypothyroidism compared versus $0.292 \pm 0.008$ in the control group, with a statistically significant difference between the two groups $(p<0.001)$. At baseline, rats in both groups were not obese. At the end of the study, all rats in hypothyroidism group developed obesity. Obesity was noted in only one rat in the control group. There was statistically significant difference with respect to obesity between the two groups $(p<0.001)$. 
TABLE 1: Results of biochemical parameters.

\begin{tabular}{lccc}
\hline & $\begin{array}{c}\text { Hypothyroidism } \\
(n=8)\end{array}$ & $\begin{array}{c}\text { Controls } \\
(n=8)\end{array}$ & $p^{*}$ \\
\hline TSH $(\mathrm{mIU} / \mathrm{L})$ & $2.16 \pm 0.35$ & $0.84 \pm 0.06$ & $<\mathbf{0 . 0 0 1}$ \\
fT4 (pmol/L) & $10.33 \pm 4.46$ & $16.07 \pm 2.19$ & $\mathbf{0 . 0 0 6}$ \\
fT3 (nmol/L) & $5.32 \pm 0.62$ & $7.47 \pm 0.12$ & $>\mathbf{0 . 0 0 1}$ \\
Visfatin (ng/mL) & $88.04 \pm 7.29$ & $89.67 \pm 5.92$ & $\mathbf{0 . 0 5}$ \\
Fasting plasma glucose (mg/dL) & $126.25 \pm 23.4$ & $102.63 \pm 15.51$ & $\mathbf{0 . 0 3 2}$ \\
ALT (U/L) & $58.38 \pm 22.9$ & $38.75 \pm 6.04$ & $\mathbf{0 . 0 3 4}$ \\
AST (U/L) & $153.63 \pm 35.1$ & $98.5 \pm 17.65$ & $\mathbf{0 . 0 0 1}$ \\
Triglyceride (mg/dL) & $38.5 \pm 6.89$ & $37.63 \pm 11.36$ & $23 \pm 1.93$ \\
HDL (mg/dL) & $24.5 \pm 3.34$ & $35.88 \pm 3.8$ & $>0.05$ \\
LDL (mg/dL) & $53 \pm 10.85$ & $1 \pm 0.36$ \\
Fasting insulin levels $(\mathrm{mU} / \mathrm{mL})$ & $1.19 \pm 0.5$ & & $\mathbf{0 . 0 0 1}$ \\
\hline
\end{tabular}

${ }^{*}$ Student's $t$-test was used.

3.2. Evaluation of Biochemical Parameters. TSH in the hypothyroidism group was $2.16 \pm 0.35 \mathrm{mIU} / \mathrm{L}$ and differed statistically significantly from the control group $(p<0.001)$. fT4 in the hypothyroidism group was $10.33 \pm 4.46 \mathrm{pmol} / \mathrm{L}$, which was statistically significantly lower compared to the control group ( $p=0.006$ ). Similarly, fT3 in the hypothyroidism group was $5.32 \pm 0.62 \mathrm{nmol} / \mathrm{L}$ and was statistically significantly lower compared to the control group $(p<$ 0.001 ). The two groups did not differ statistically significantly with respect to visfatin levels $(p>0.05)$. All biochemical parameters are provided in Table 1.

\subsection{Pathology Results}

3.3.1. Macroscopic Results. In all hypothyroidism-induced rats, livers appeared pale and brittle. No macroscopic change was observed in the livers of the rats in the control group.

3.3.2. Microscopic Histopathologic Results. Livers of all hypothyroidism-induced rats involved mild steatosis. No microscopic steatosis was observed in the livers of the rats in the control group. There was statistically significant difference with respect to microscopic hepatosteatosis between the two groups $(p<0.001)$. With HE staining, in livers of all hypothyroidism-induced rats, clear microvesicular vacuoles with sharp edges affecting hepatocyte to varying degrees and distributed randomly were seen (Figure 1). The vacuoles were determined to be fat vacuoles with Oil Red staining (Figure 2). Nuclei of hepatocytes were of normal appearance and location. However, impaired structure of remark cords and mild parenchymal degeneration were the other observed changes.

With Oil Red staining in the livers of the rats in the control group, there were hardly remarkable, randomly distributed, sporadic microvesicular fat vacuoles. Both the hypothyroidism group and the control group had portally located mild mononuclear cell infiltrations consisting of lymphocytes and macrophages.

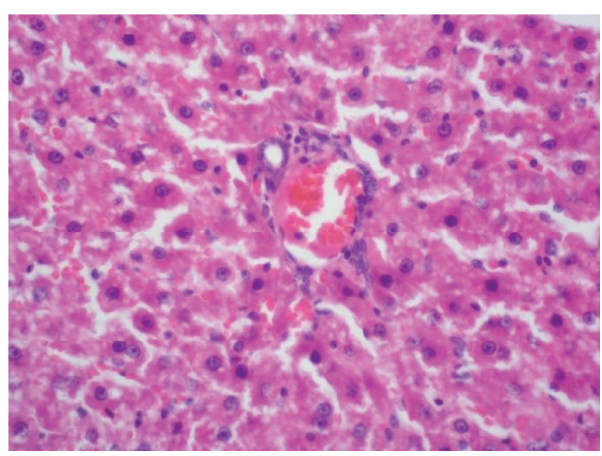

FIGURE 1: With HE staining, clear microvesicular vacuoles with sharp edges affecting hepatocyte to varying degrees and distributed randomly were seen.

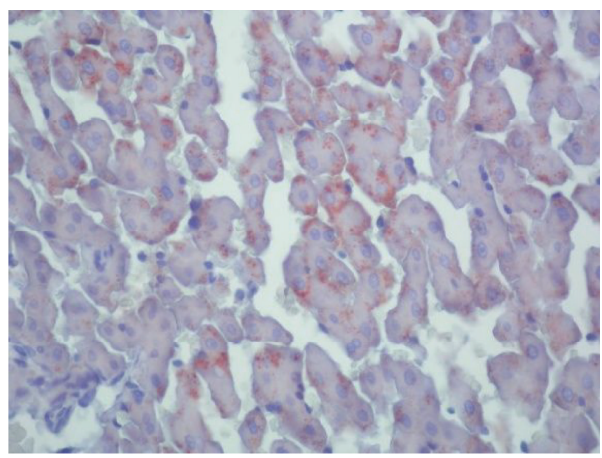

FIGURE 2: The vacuoles were determined to be fat vacuoles with Oil Red staining.

\section{Discussion}

In our study, we detected mild hepatosteatosis in all hypothyroidism-induced rats. Randomly distributed sporadic fat vacuoles observed in the livers of rats in the healthy control group can normally be found in rat livers [17]. The number 
of affected hepatocytes and the severity of fat vacuoles buildup in the livers of hypothyroidism-induced rats indicate that hypothyroidism leads to fatty changes in the liver. Portally located mild mononuclear cell accumulations in both hypothyroidism-induced and control groups are changes with unknown relevance that can be seen in rat livers [17].

A study by Liangpunsakul and Chalasani [18] included 174 patients with NASH diagnosed with liver biopsy. Hypothyroidism was detected in $15 \%$ of the patients with NASH and in $7.2 \%$ of the control group. The authors suggested that hypothyroidism was more prevalent among NASH patients compared to the control group. In a study by Silveira et al. [19], frequency of thyroid dysfunction was $13 \%$ in patients with primary biliary cirrhosis, $11 \%$ in patients with primary sclerosing cholangitis, and $25 \%$ in patients with NAFLD and $20 \%$ of NAFLD patients had hypothyroidism. In the sectional study by Pagadala et al. [20], 21\% of the patients were diagnosed with NASH/NAFLD with liver biopsy and $25 \%$ of non-NASH patients were diagnosed with NAFLD and $12.8 \%$ of the healthy control group had hypothyroidism. The authors reported a higher prevalence of hypothyroidism in NAFLD patients compared to the control group. In a study by Parikh et al. [21] in Western India, $16.8 \%$ of the patients with USG-diagnosed NAFLD had hypothyroidism, which was significantly higher compared to the control group. Hypothyroidism was underlined as a risk factor independent from the known risk factors for NAFLD. Mazo et al. [22] found the prevalence of hypothyroidism in NAFLD patients as $15.5 \%$ compared to $15.7 \%$ in NASH patients and $15.2 \%$ in patients with steatosis. In the study by Carulli et al. [10] liver biopsies demonstrated NAFLD in 69 patients, MASH in 44 patients, and steatosis in 25 patients. Patients with NASH had significantly higher TSH levels compared to patients with steatosis, and the authors emphasized that TSH level can be an independent positive risk factor for NASH. Xu et al. [23] monitored 63 patients with subclinical hypothyroidism and 35 euthyroid healthy control groups for a median of 4.92 years for NAFLD development. The incidence of NAFLD development in patients with subclinical hypothyroidism was significantly higher than in euthyroid individuals.

However, unlike these studies, there are studies demonstrating a lack of relationship between hypothyroidism and NASH/NAFLD. In a study by Ittermann et al. [12], no relationship was found between hepatosteatosis and hypothyroidism. Hepatosteatosis was not related with TSH or fT3 concentrations but there was a relationship with low fT4 concentrations. A study by Eshraghian et al. [11] did not determine a correlation between NAFLD and thyroid dysfunction. $\mathrm{TSH}, \mathrm{fT} 4$, and $\mathrm{fT} 3$ were not statistically different in patients with and without NAFLD.

Our literature scan did not yield a study evaluating hepatosteatosis, NAFLD development with liver biopsy in patients with hypothyroidism. In our study, we histopathologically evaluated if hypothyroidism leads to hepatosteatosis, NAFLD development. Because liver biopsy would be an invasive procedure to be used in hypothyroidism patients, our study used an animal model. We histopathologically determined with this study that hypothyroidism leads to mild hepatosteatosis in rats.
We believe that many factors are involved in explaining the mechanism of the relationship between hypothyroidism and NAFLD. As one of these factors, obesity is commonly seen together with NAFLD. In morbidly obese patients, NAFLD frequency is as high as $90 \%$ and advanced disease (e.g., NASH) is seen in 9 to $40 \%$. The relationship between BMI and the extent of fat build-up and hepatic damage has been shown in a number of studies [24-26]. In obese cohorts scheduled to receive bariatric surgery, it was reported that steatohepatitis prevalence could be from $37 \%$ to as high as 91\% with biopsy [27].

Studies supporting the relationship between hypothyroidism and NAFLD emphasize that this relationship may be associated with obesity development secondary to hypothyroidism [28, 29].

Eshraghian et al. [11] found a relationship between central obesity and NAFLD but not between hypothyroidism and NAFLD. In our study, all hypothyroidism-induced rats developed obesity according to the Lee index. Obesity was observed in only one rat in the healthy control group. There was a statistically significant difference between the two groups with respect to obesity frequency. We believe that obesity is one of the major determinants in the relationship between hypothyroidism and NAFLD.

Thyroid hormones mediate the effects on lipid metabolism via thyroid hormone receptor $\beta$ expressed from the liver [30]. Hypothyroidism can also lead to increased serum triglyceride levels by decreasing hepatic lipoprotein lipase activity [31]. Several studies demonstrated increased LDL cholesterol levels in subclinical hypothyroidism patients [4, 32-34].

Fabbrini et al. [35] found higher plasma VLDL and triglyceride concentrations in subclinical hypothyroidism patients compared to euthyroid individuals. Several studies demonstrated a negative correlation between triglyceride levels and fT4 levels [36, 37]. Hypertriglyceridemia is known as a factor that contributes to NAFLD development $[38,39]$. Lipid profiles of patients diagnosed with NAFLD/NASH involve significant increase in LDL cholesterol and triglyceride levels [40]. In an animal study by Cable et al. [41], in which a hepatosteatosis model was induced, the animals were given liver-targeted thyroid hormone receptor agonist. The study demonstrated that treatment with liver-targeted thyroid hormone receptor agonist in animals with coexisting NASH and hyperlipidemia decreased LDL cholesterol and could provide additional therapeutic benefits.

In our study, the LDL level of hypothyroidism-induced group was statistically significantly higher compared to the healthy control group. The groups were not different with regard to serum triglyceride and HDL levels. We believe that this change in the lipid profile supports NAFLD development in the hypothyroidism-induced group.

Most NAFLD patients have insulin resistance and diabetes mellitus usually accompanies this [42]. Liangpunsakul and Chalasani [18] determined that $38.5 \%$ of the patients with NAFLD had diabetes mellitus. Pagadala et al. [20] found diabetes mellitus in $42.7 \%$ of the patients with NAFLD, which was significantly higher compared to the control group. It has been reported that hypothyroidism may be associated with 
insulin resistance, which may be decreased if hypothyroidism is treated [43]. Smithson [44] determined that hypothyroidism was more common in diabetic patients compared to overall population. Gronich et al. [45] concluded that hypothyroidism was a risk factor for diabetes mellitus. The authors emphasized that the risk of diabetes mellitus could be reduced by identifying and treating hypothyroidism. In our study, mean fasting blood glucose was $126.25 \pm 23.4 \mathrm{mg} / \mathrm{dL}$ in hypothyroidism-induced group and $102.63 \pm 15.51 \mathrm{mg} / \mathrm{dL}$ in the control group, with a statistically significant difference between the groups ( $p=0.032)$. Our results supported that hypothyroidism increases the risk of diabetes mellitus. Pronounced hyperglycemia in the hypothyroidism-induced group was considered as one of the consequences that support NAFLD development.

The role of adipokines in NAFLD pathogenesis was demonstrated by previous studies [46, 47]. Visfatin, an adipokine, is a hormone, plasma levels of which are also associated with obesity, visceral fat, type 2 diabetes, and metabolic syndrome [48]. High levels of visfatin were found in obesity, type 2 diabetes, and metabolic syndrome $[49,50]$. There is limited data on the relationship between NAFLD and visfatin levels. Lower visfatin levels were observed in patients with NASH compared to those with NAFLD $[47,51]$. Several studies attempted to describe the mechanism between hypothyroidism and NAFLD through adipokines. Some of these studies identified no relationship between adipokines and hypothyroidism $[52,53]$. Contradicting views exist on the relationship between visfatin and hypothyroidism. One study found a positive correlation between TSH and visfatin levels and negative correlation between fT3, fT4, and visfatin levels [54]. Another study observed higher visfatin levels in both subjects with hyperthyroidism and those with hypothyroidism compared to controls and found increased visfatin levels following treatment [55]. There are studies with contradicting results on the role of thyroid hormones on visfatin regulation [56, 57]. Ozkaya et al. [54] argued that the effect of hyperthyroidism on several metabolic parameters could be mediated by visfatin although the effect of thyroid dysfunction on the production and release of adipocytokines has not been fully understood. In our study, the two groups did not differ in visfatin levels. Our study supports the view that visfatin is not involved in the development of NAFLD secondary to hypothyroidism.

\section{Conclusion}

We found that hypothyroidism-induced rats had mild hepatosteatosis as opposed to the control group histopathologically. Our study indicates that hypothyroidism can cause NAFLD.

\section{Competing Interests}

The authors declare that they have no competing interests.

\section{Acknowledgments}

This research was supported by Scientific Research Projects of Adnan Menderes University (Project no. TPF-15011).

\section{References}

[1] M. A. Michalaki, A. G. Vagenakis, A. S. Leonardou et al., "Thyroid function in humans with morbid obesity," Thyroid, vol. 16, no. 1, pp. 73-78, 2006.

[2] Y. Raftopoulos, D. J. Gagné, P. Papasavas et al., "Improvement of hypothyroidism after laparoscopic Roux-en-Y gastric bypass for morbid obesity," Obesity Surgery, vol. 14, no. 4, pp. 509-513, 2004.

[3] T. Tzotzas, G. E. Krassas, T. Konstantinidis, and M. Bougoulia, "Changes in lipoprotein(a) levels in overt and subclinical hypothyroidism before and during treatment," Thyroid, vol. 10, no. 9, pp. 803-808, 2000.

[4] Z. Efstathiadou, S. Bitsis, H. J. Milionis et al., "Lipid profile in subclinical hypothyroidism: is L-thyroxine substitution beneficial?" European Journal of Endocrinology, vol. 145, no. 6, pp. 705-710, 2001.

[5] K. Te Sligte, I. Bourass, J. P. Sels, A. Driessen, R. W. Stockbrugger, and G. H. Koek, "Non-alcoholic steatohepatitis: review of a growing medical problem," European Journal of Internal Medicine, vol. 15, no. 1, pp. 10-21, 2004.

[6] S. K. Erickson, "Nonalcoholic fatty liver disease," Journal of Lipid Research, vol. 50, supplement, pp. 412-416, 2009.

[7] G. Pagano, G. Pacini, G. Musso et al., "Nonalcoholic steatohepatitis, insulin resistance, and metabolic syndrome: further evidence for an etiologic association," Hepatology, vol. 35, no. 2, pp. 367-372, 2002.

[8] A. Eshraghian and A. H. Jahromi, "Non-alcoholic fatty liver disease and thyroid dysfunction: a systematic review," World Journal of Gastroenterology, vol. 20, no. 25, pp. 8102-8109, 2014.

[9] J. Zhang, H. Sun, L. Chen et al., "Relationship between serum TSH level with obesity and NAFLD in euthyroid subjects," Journal of Huazhong University of Science and Technology: Medical Science, vol. 32, no. 1, pp. 47-52, 2012.

[10] L. Carulli, S. Ballestri, A. Lonardo et al., "Is nonalcoholic steatohepatitis associated with a high-though-normal thyroid stimulating hormone level and lower cholesterol levels?" Internal and Emergency Medicine, vol. 8, no. 4, pp. 297-305, 2013.

[11] A. Eshraghian, M. H. Dabbaghmanesh, H. Eshraghian, M. R. Fattahi, and G. R. Omrani, "Nonalcoholic fatty liver disease in a cluster of Iranian population: thyroid status and metabolic risk factors," Archives of Iranian Medicine, vol. 16, no. 10, pp. 584$589,2013$.

[12] T. Ittermann, R. Haring, H. Wallaschofski et al., "Inverse association between serum free thyroxine levels and hepatic steatosis: results from the study of health in pomerania," Thyroid, vol. 22, no. 6, pp. 568-574, 2012.

[13] G. E. Chung, D. Kim, W. Kim et al., "Non-alcoholic fatty liver disease across the spectrum of hypothyroidism," Journal of Hepatology, vol. 57, no. 1, pp. 150-156, 2012.

[14] C. MacNabb, E. O'Hare, J. Cleary, and A. P. Georgopoulos, "Varied duration of congenital hypothyroidism potentiates perseveration in a response alternation discrimination task," Neuroscience Research, vol. 36, no. 2, pp. 121-127, 2000.

[15] L. L. Bernardis and B. D. Patterson, "Correlation between "Lee index" and carcass fat content in weanling and adult female rats with hypothalamic lesions," Journal of Endocrinology, vol. 40, no. 4, pp. 527-528, 1968.

[16] C. Ruehl-Fehlert, B. Kittel, G. Morawietz et al., "Revised guides for organ sampling and trimming in rats and mice-part 1: a joint publication of the RITA and NACAD groups," Experimental and Toxicologic Pathology, vol. 55, no. 2-3, pp. 91-106, 2003. 
[17] C. L. Scudamore, "Acquiring, recording, and analyzing pathology data from experimental mice: an overview," Current Protocols in Mouse Biology, vol. 4, no. 1, pp. 1-10, 2014.

[18] S. Liangpunsakul and N. Chalasani, "Is hypothyroidism a risk factor for non-alcoholic steatohepatitis?" Journal of Clinical Gastroenterology, vol. 37, no. 4, pp. 340-343, 2003.

[19] M. G. Silveira, F. D. Mendes, N. N. Diehl, F. T. Enders, and K. D. Lindor, "Thyroid dysfunction in primary biliary cirrhosis, primary sclerosing cholangitis and non-alcoholic fatty liver disease," Liver International, vol. 29, no. 7, pp. 1094-1100, 2009.

[20] M. R. Pagadala, C. O. Zein, S. Dasarathy, L. M. Yerian, R. Lopez, and A. J. McCullough, "Prevalence of hypothyroidism in nonalcoholic fatty liver disease," Digestive Diseases and Sciences, vol. 57, no. 2, pp. 528-534, 2012.

[21] P. Parikh, A. Phadke, and P. Sawant, "Prevalence of hypothyroidism in nonalcoholic fatty liver disease in patients attending a tertiary hospital in western India," Indian Journal of Gastroenterology, vol. 34, no. 2, pp. 169-173, 2015.

[22] D. F. D. C. Mazo, V. M. R. de Lima, J. T. Stefano, F. Rabelo, J. Faintuch, and C. P. de Oliveira, "Gluco-lipidic indices in treated hypothyroidism associated with nonalcoholic fatty liver disease," Arquivos de Gastroenterologia, vol. 48, no. 3, pp. 186189, 2011.

[23] L. Xu, H. Ma, M. Miao, and Y. Li, "Impact of subclinical hypothyroidism on the development of non-alcoholic fatty liver disease: a prospective case-control study," Journal of Hepatology, vol. 57, no. 5, pp. 1153-1154, 2012.

[24] J.-W. Park, G. Jeong, S. J. Kim, M. K. Kim, and S. M. Park, "Predictors reflecting the pathological severity of non-alcoholic fatty liver disease: comprehensive study of clinical and immunohistochemical findings in younger Asian patients," Journal of Gastroenterology and Hepatology, vol. 22, no. 4, pp. 491-497, 2007.

[25] M. Moretto, C. Kupski, C. C. Mottin et al., "Hepatic steatosis in patients undergoing bariatric surgery and its relationship to body mass index and co-morbidities," Obesity Surgery, vol. 13, no. 4, pp. 622-624, 2003.

[26] P.-J. Hsiao, K.-K. Kuo, S.-J. Shin et al., "Significant correlations between severe fatty liver and risk factors for metabolic syndrome," Journal of Gastroenterology and Hepatology, vol. 22, no. 12, pp. 2118-2123, 2007.

[27] M. Machado, P. Marques-Vidal, and H. Cortez-Pinto, "Hepatic histology in obese patients undergoing bariatric surgery," Journal of Hepatology, vol. 45, no. 4, pp. 600-606, 2006.

[28] N. Knudsen, P. Laurberg, L. B. Rasmussen et al., "Small differences in thyroid function may be important for body mass index and the occurrence of obesity in the population," Journal of Clinical Endocrinology and Metabolism, vol. 90, no. 7, pp. 4019-4024, 2005.

[29] A. Nyrnes, R. Jorde, and J. Sundsfjord, "Serum TSH is positively associated with BMI," International Journal of Obesity, vol. 30, no. 1, pp. 100-110, 2006.

[30] A. J. Hulbert, "Thyroid hormones and their effects: a new perspective," Biological Reviews of the Cambridge Philosophical Society, vol. 75, no. 4, pp. 519-631, 2000.

[31] L. H. Duntas, “Thyroid disease and lipids," Thyroid, vol. 12, no. 4, pp. 287-293, 2002.

[32] L. Tian, C. Gao, J. Liu, and X. Zhang, "Increased carotid arterial stiffness in subclinical hypothyroidism," European Journal of Internal Medicine, vol. 21, no. 6, pp. 560-563, 2010.
[33] M. Unubol, U. Eryilmaz, E. Guney, C. Akgullu, and I. K. Omurlu, "Epicardial adipose tissue in patients with subclinical hypothyroidism," Minerva Endocrinologica, vol. 39, no. 2, pp. 135-140, 2014.

[34] E. E. Türemen, B. Çetinarslan, T. Şahin, Z. Cantürk, and I. Tarkun, "Endothelial dysfunction and low grade chronic inflammation in subclinical hypothyroidism due to autoimmune thyroiditis," Endocrine Journal, vol. 58, no. 5, pp. 349-354, 2011.

[35] E. Fabbrini, F. Magkos, B. W. Patterson, B. Mittendorfer, and S. Klein, "Subclinical hypothyroidism and hyperthyroidism have opposite effects on hepatic very low density lipoprotein triglyceride kinetics," The Journal of Clinical Endocrinology \& Metabolism, vol. 97, no. 3, pp. 414-418, 2012.

[36] S.-Y. Lin, Y.-Y. Wang, P.-H. Liu, W.-A. Lai, and W. H.-H. Sheu, "Lower serum free thyroxine levels are associated with metabolic syndrome in a Chinese population," Metabolism: Clinical and Experimental, vol. 54, no. 11, pp. 1524-1528, 2005.

[37] H. S. Shon, E. D. Jung, S. H. Kim, and J. H. Lee, "Free T4 is negatively correlated with body mass index in euthyroid women," Korean Journal of Internal Medicine, vol. 23, no. 2, pp. 53-57, 2008.

[38] I. J. Goldberg and H. N. Ginsberg, "Ins and outs modulating hepatic triglyceride and development of nonalcoholic fatty liver disease," Gastroenterology, vol. 130, no. 4, pp. 1343-1346, 2006.

[39] P. Puri, R. A. Baillie, M. M. Wiest et al., "A lipidomic analysis of nonalcoholic fatty liver disease," Hepatology, vol. 46, no. 4, pp. 1081-1090, 2007.

[40] G. Musso, R. Gambino, and M. Cassader, "Recent insights into hepatic lipid metabolism in non-alcoholic fatty liver disease (NAFLD)," Progress in Lipid Research, vol. 48, no. 1, pp. 1-26, 2009.

[41] E. E. Cable, P. D. Finn, J. W. Stebbins et al., "Reduction of hepatic steatosis in rats and mice after treatment with a liver-targeted thyroid hormone receptor agonist," Hepatology, vol. 49, no. 2, pp. 407-417, 2009.

[42] L. L. Stein, M. H. Dong, and R. Loomba, "Insulin sensitizers in nonalcoholic fatty liver disease and steatohepatitis: current status," Advances in Therapy, vol. 26, no. 10, pp. 893-907, 2009.

[43] I. Kowalska, J. Borawski, A. Nikołajuk et al., "Insulin sensitivity, plasma adiponectin and sICAM-1 concentrations in patients with subclinical hypothyroidism: response to levothyroxine therapy," Endocrine, vol. 40, no. 1, pp. 95-101, 2011.

[44] M. J. Smithson, "Screening for thyroid dysfunction in a community population of diabetic patients," Diabetic Medicine, vol. 15, no. 2, pp. 148-150, 1998.

[45] N. Gronich, S. N. Deftereos, I. Lavi, A. S. Persidis, D. R. Abernethy, and G. Rennert, "Hypothyroidism is a risk factor for newonset diabetes: a cohort study," Diabetes Care, vol. 38, no. 9, pp. 1657-1664, 2015.

[46] G. Musso, R. Gambino, M. Durazzo et al., "Adipokines in NASH: postprandial lipid metabolism as a link between adiponectin and liver disease," Hepatology, vol. 42, no. 5, pp. 11751183, 2005.

[47] M. H. Jarrar, A. Baranova, R. Collantes et al., "Adipokines and cytokines in non-alcoholic fatty liver disease," Alimentary Pharmacology and Therapeutics, vol. 27, no. 5, pp. 412-421, 2008.

[48] D. G. Haider, G. Schaller, S. Kapiotis, C. Maier, A. Luger, and M. Wolzt, "The release of the adipocytokine visfatin is regulated by glucose and insulin," Diabetologia, vol. 49, no. 8, pp. 1909-1914, 2006. 
[49] M.-P. Chen, F.-M. Chung, D.-M. Chang et al., "Elevated plasma level of visfatin/pre-B cell colony-enhancing factor in patients with type 2 diabetes mellitus," Journal of Clinical Endocrinology and Metabolism, vol. 91, no. 1, pp. 295-299, 2006.

[50] D. G. Haider, K. Schindler, G. Schaller, G. Prager, M. Wolzt, and B. Ludvik, "Increased plasma visfatin concentrations in morbidly obese subjects are reduced after gastric banding," Journal of Clinical Endocrinology and Metabolism, vol. 91, no. 4, pp. 1578-1581, 2006.

[51] R. Aller, D. A. de Luis, O. Izaola et al., "Influence of visfatin on histopathological changes of non-alcoholic fatty liver disease," Digestive Diseases and Sciences, vol. 54, no. 8, pp. 1772-1777, 2009.

[52] A. E. Altinova, F. B. Törüner, M. Aktürk et al., "Adiponectin levels and cardiovascular risk factors in hypothyroidism and hyperthyroidism," Clinical Endocrinology, vol. 65, no. 4, pp. 530-535, 2006.

[53] C. N. Aragão, L. L. Souza, A. Cabanelas, K. J. Oliveira, and C. C. Pazos-Moura, "Effect of experimental hypo- and hyperthyroidism on serum adiponectin," Metabolism, vol. 56, no. 1, pp. 6-11, 2007.

[54] M. Ozkaya, M. Sahin, E. Cakal et al., "Visfatin plasma concentrations in patients with hyperthyroidism and hypothyroidism before and after control of thyroid function," Journal of Endocrinological Investigation, vol. 32, no. 5, pp. 435-439, 2009.

[55] A. Caixàs, R. Tirado, J. Vendrell et al., "Plasma visfatin concentrations increase in both hyper and hypothyroid subjects after normalization of thyroid function and are not related to insulin resistance, anthropometric or inflammatory parameters," Clinical Endocrinology, vol. 71, no. 5, pp. 733-738, 2009.

[56] M. Tanaka, M. Nozaki, A. Fukuhara et al., "Visfatin is released from 3T3-L1 adipocytes via a non-classical pathway," Biochemical and Biophysical Research Communications, vol. 359, no. 2, pp. 194-201, 2007.

[57] R. MacLaren, W. Cui, and K. Cianflone, "Visfatin expression is hormonally regulated by metabolic and sex hormones in 3T3-L1 pre-adipocytes and adipocytes," Diabetes, Obesity \& Metabolism, vol. 9, no. 4, pp. 490-497, 2007. 


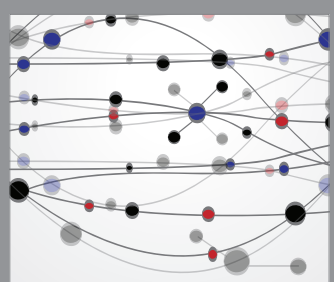

The Scientific World Journal
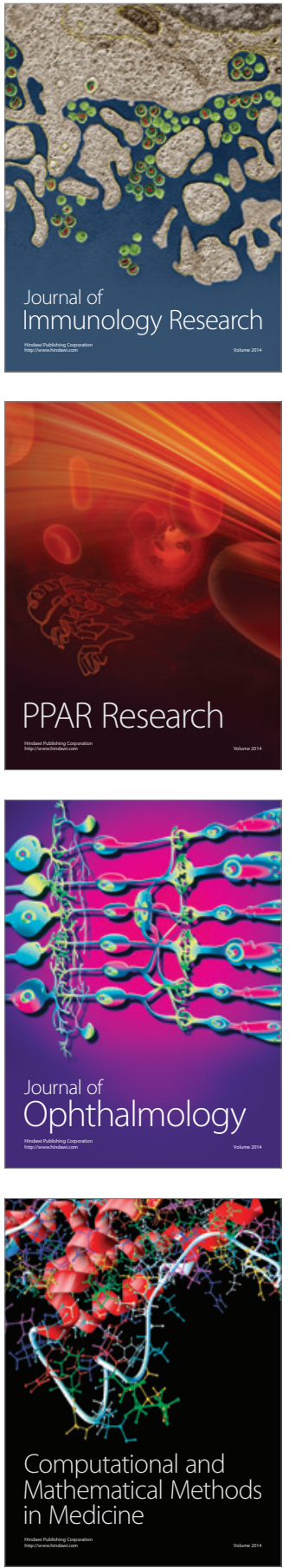

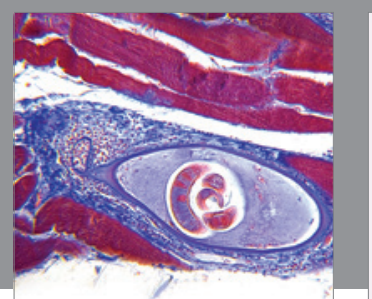

Gastroenterology Research and Practice

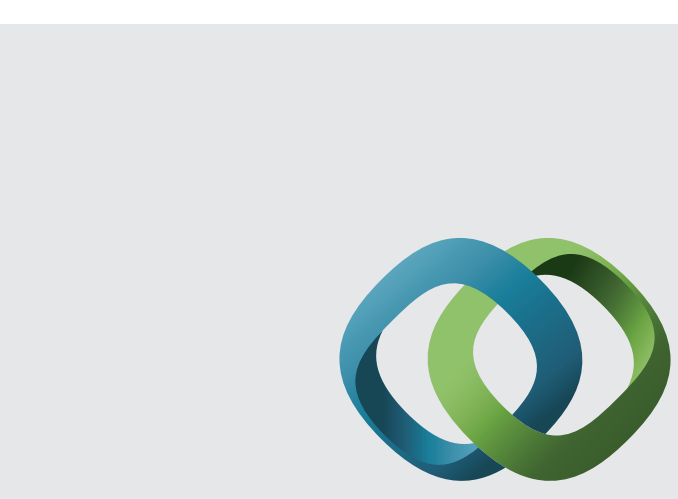

\section{Hindawi}

Submit your manuscripts at

http://www.hindawi.com
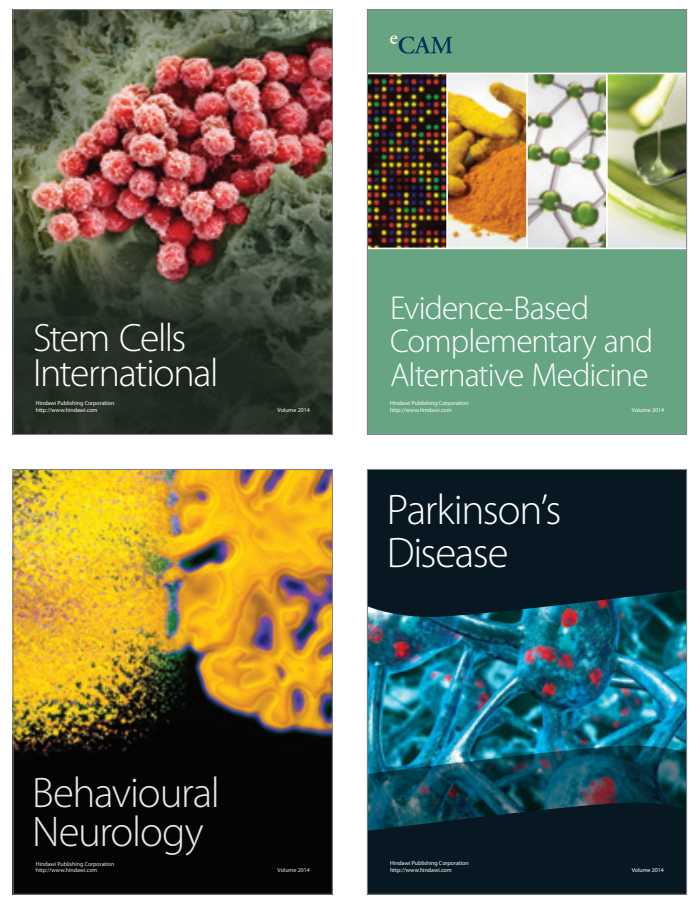
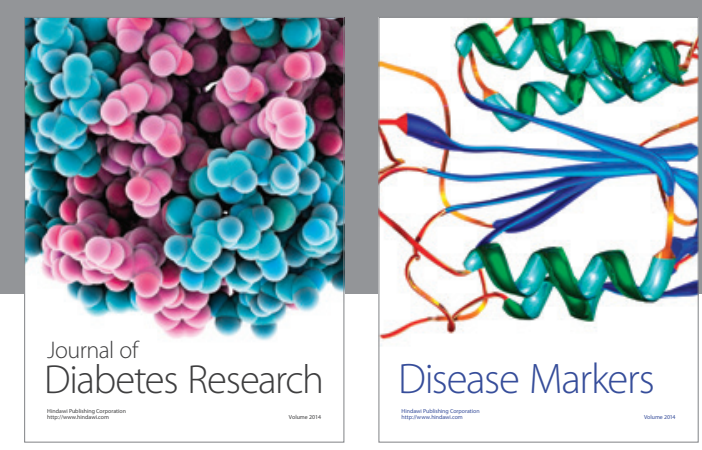

Disease Markers
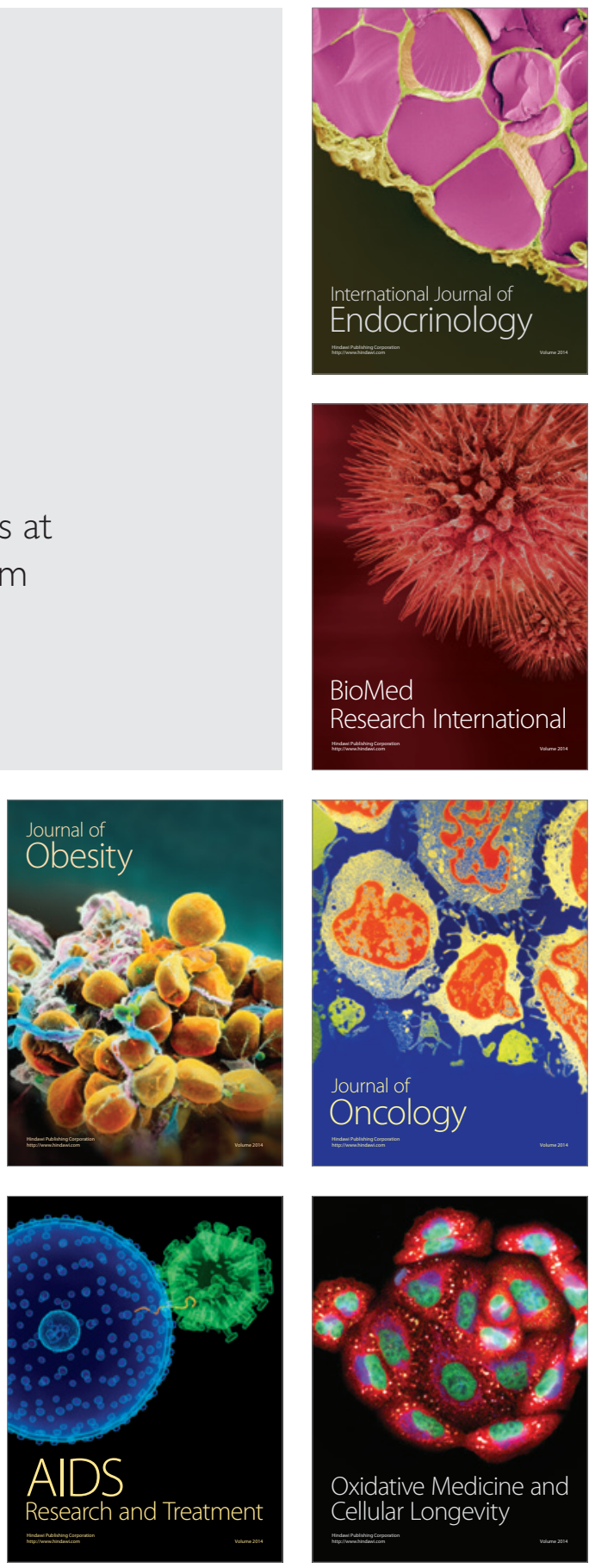